\title{
Predicting acute radiation induced xerostomia in head and neck Cancer using MR and CT Radiomics of parotid and submandibular glands
}

Khadija Sheikh, Sang Ho Lee, Zhi Cheng, Pranav Lakshminarayanan, Luke Peng, Peijin Han, Todd R. McNutt, Harry Quon and Junghoon Lee* (D)

\begin{abstract}
Purpose: To analyze baseline CT/MR-based image features of salivary glands to predict radiation-induced xerostomia 3-months after head-and-neck cancer (HNC) radiotherapy.

Methods: A retrospective analysis was performed on 266 HNC patients who were treated using radiotherapy at our institution between 2009 and 2018. CT and T1 post-contrast MR images along with NCI-CTCAE xerostomia grade (3month follow-up) were prospectively collected at our institution. CT and MR images were registered on which parotid/ submandibular glands were contoured. Image features were extracted for ipsilateral/contralateral parotid and submandibular glands relative to the location of the primary tumor. Dose-volume-histogram $(\mathrm{DVH})$ parameters were also acquired. Features were pre-selected based on Spearman correlation before modelling by examining the correlation with xerostomia $(p<0.05)$. A shrinkage regression analysis of the pre-selected features was performed using LASSO. The internal validity of the variable selection was estimated by repeating the entire variable selection procedure using a leave-one-out-cross-validation. The most frequently selected variables were considered in the final model. A generalized linear regression with repeated ten-fold cross-validation was developed to predict radiation-induced xerostomia at 3-months after radiotherapy. This model was tested in an independent dataset $(n=50)$ of patients who were treated at the same institution in 2017-2018. We compared the prediction performances under eight conditions (DVH-only, CT-only, MR-only, $C T+M R, D V H+C T$, DVH $+C T+M R$, Clinical+CT + MR, and Clinical+DVH + CT +MR) using the area under the receiver operating characteristic curve (ROC-AUC).

Results: Among extracted features, $7 \subset T, 5 \mathrm{MR}$, and $2 \mathrm{DVH}$ features were selected. The internal cohort $(n=216)$ ROC-AUC values for DVH, CT, MR, and Clinical+DVH +CT + MR features were $0.73 \pm 0.01,0.69 \pm 0.01,0.70 \pm 0.01$, and $0.79 \pm 0.01$, respectively. The validation cohort $(n=50) \mathrm{ROC}-\mathrm{AUC}$ values for $\mathrm{DVH}, \mathrm{CT}, \mathrm{MR}$, and Clinical+DVH $+\mathrm{CT}+\mathrm{MR}$ features were $0.63,0.57,0.66$, and 0.68 , respectively. The DVH-ROC was not significantly different than the CT-ROC $(p=0.8)$ or MR-ROC $(p=0.4)$. However, the $C T+M R-R O C$ was significantly different than the CT-ROC $(p=0.03)$, but not the Clinical+DVH + $C T+$ MR model $(p=0.5)$.

Conclusion: Our results suggest that baseline $C T$ and MR image features may reflect baseline salivary gland function and potential risk for radiation injury. The integration of baseline image features into prediction models has the potential to improve xerostomia risk stratification with the ultimate goal of truly personalized HNC radiotherapy.
\end{abstract}

Keywords: Head and neck cancer, Radiation therapy, Radiomics, Machine learning, Xerostomia

\footnotetext{
*Correspondence: junghoon@jhu.edu

Department of Radiation Oncology and Molecular Radiation Sciences, Johns

Hopkins University School of Medicine, 401 North Broadway, Suite 1440,

Baltimore, MD 21287-5678, USA
}

(c) The Author(s). 2019 Open Access This article is distributed under the terms of the Creative Commons Attribution 4.0 International License (http://creativecommons.org/licenses/by/4.0/), which permits unrestricted use, distribution, and reproduction in any medium, provided you give appropriate credit to the original author(s) and the source, provide a link to the Creative Commons license, and indicate if changes were made. The Creative Commons Public Domain Dedication waiver (http://creativecommons.org/publicdomain/zero/1.0/) applies to the data made available in this article, unless otherwise stated. 


\section{Background}

Radiation therapy (RT), often with concurrent chemotherapy, is frequently used in the management of head and neck cancer (HNC) as definitive or adjuvant treatment. RT for HNC improves local control but is associated with significant treatment-related toxicities such as xerostomia [1, 2]. Approximately $50-80 \%$ of patients with $\mathrm{HNC}$ will experience xerostomia to some degree after RT [3, 4]. While these swallow-related toxicities significantly influence long-term patient outcomes and quality of life, our ability to robustly characterize these complications as they relate to individual patients and the radiotherapy dosimetry delivered to salivary glands is limited.

In radiation oncology, there is increasing popularity for rapid-learning health systems which use routine clinical data to develop models that can be used to predict patient specific treatment outcomes [5-7]. In addition to predicting outcomes, the goal of decision support systems is to improve overall patient care and determine when and how to personalize patients' treatments. Machine learning algorithms have emerged as popular tools for decision support. These algorithms are already being applied to many aspects of radiation therapy including: target delineation $[8,9]$, treatment planning $[10,11]$, radiation physics quality assurance [12], and outcome [13] and tumor response modelling [14]. With recent advancements in image processing, informatics, and machine learning, medical imaging is increasingly being used for improved clinical decision making. Studies have demonstrated that the variability in clinical image intensity, shape, and texture can be quantified generating a radiomic signature for individual tumors and normal anatomic structures [15-20]. For radiation therapy, radiomics offers the potential to significantly influence clinical decision-making, therapy planning, and followup workflow. In $\mathrm{HNC}$, a radiomic signature has been shown to be prognostic and has been validated across several institutions $[19,20]$. Radiomics derived from computed tomography $(\mathrm{CT})$ have also been used to predict xerostomia and survival in HNC patients [18, 21, 22].

To our knowledge, the incorporation of MR-based biomarkers with CT and dosimetry features in acute RT-induced xerostomia prediction models has not been investigated in HNC. Thus, the objective of this study was to analyze baseline CT/MR image features of salivary glands to better understand their role in the prediction of radiation-induced xerostomia 3 months after HNC radiotherapy. We hypothesized that baseline $\mathrm{CT} / \mathrm{MR}$ image features are related to xerostomia and incorporating these into a prediction model improves the accuracy of predicting radiationinduced xerostomia compared to dosimetric information alone.

\section{Materials and methods}

\section{Patients}

HNC patients treated at Johns Hopkins Hospital who underwent intensity modulated radiotherapy (IMRT) from 2009 through 2018 (on a protocol for retrospective data analysis approved by the institutional review board) were included. Patients who did not have MR images were excluded. NCI-CTCAE v4.0 xerostomia grade was assessed by physicians at the point of care at 3 months post-RT. Moderate to severe xerostomia incidence was defined as grade $\geq 2$, compared to the reference group which was defined as xerostomia grade 0 or 1 . All patients were treated with either IMRT, VMAT, or TomoTherapy. All treatments included a simultaneous integrated boost and attempted to spare dose to the parotid glands and swallowing structures without compromising the dose to the target volumes.

\section{Image data}

All images were acquired at the time of simulation, prior to the start of treatment. For both training and validation sets, T1-weighted MRI was acquired using Siemens Magnetom Espree 1.5 T scanner (Siemens Medical Systems, Erlangen, Germany) with a turbo spin echo sequence post-Gd administration $(\mathrm{TE}=8.9 \mathrm{~ms}, \mathrm{TR}=577$ ms, flip angle $=150^{\circ}$, matrix size $=256 \times 256$, pixel size ranged from $0.8 \times 0.8 \mathrm{~mm}^{2}$ to $1.1 \times 1.1 \mathrm{~mm}^{2}$ depending on the field of view defined at simulation, and slice thickness $=3 \mathrm{~mm}$ ). To reduce bias and improve interpretation of the image features, MR images were resampled such that the in-plane pixel size was consistently $0.89 \times 0.89 \mathrm{~mm}^{2}$, which was the size for majority of the patients. CT images were acquired using a 16-slice Philips Brilliance Big Bore scanner (Philips, Andover, MA) with tube voltage $120 \mathrm{kVp}$ and exposure of 200 mAs. Images had $512 \times 512$ pixels with a pixel size of $1.2 \times 1.2 \mathrm{~mm}^{2}$, and a slice thickness of $3 \mathrm{~mm}$. CT images with metal artifacts (most commonly caused by dental fillings and implants) were corrected using Metal Artifact Reduction for Orthopedic Implants reconstruction. However, patients with severe artifacts were excluded in the image analysis to avoid undesirable strong influence to the image features and analysis.

\section{Feature extraction}

For each patient, ipsilateral/contralateral parotid and submandibular glands (iPG, cPG, iSG, cSG) were contoured by the attending radiation oncologist. The salivary gland volumes (including combined salivary gland volumes) were determined in centimeters cubed. The tumor volume was also determined for each patient in centimeters cubed. Missing contours were automatically segmented using in-house implemented multi-atlasbased auto-segmentation software based on a GPU- 
accelerated demons deformable image registration [23] and a statistical label fusion [24]. Each auto-segmented contour was visually checked and manually corrected for any erroneously segmented regions. CT images were registered to the MR images using Velocity (V3.2.1, Varian Medical Systems Inc., Palo Alto, CA). Contours were propagated from CT images to the MR images. Each co-registration was visually verified by overlapping the registered $\mathrm{CT}$ and the target MR images focusing on the target glands as well as by overlapping the propagated contours on the target by a single observer (KS). For each derived region of interest (ROI), dose-volume histograms (DVHs) features were calculated in 5\% increments from D10 to D95.

For each segmented salivary gland ROI, highdimensional image features were extracted from both $\mathrm{CT}$ and MR images using the PyRadiomics software package accessed via the Radiomics module of 3D Slicer [25]. The ROI was analyzed as a 3D volume. A schematic showing the feature extraction process is shown in Fig. 1. CT gray level intensities were discretized at a bin width of 25. This fixed bin width resulted in 20-25 bins for CT images based on the salivary gland ROI specified. It should be noted that texture features have been shown to be affected by the bin width used to discretize image intensities [26]. Although the optimal bin width for image feature analysis has not been established, previous HNC work has used a 25 unit bin width for the evaluation of image features [27, 28]. A fixed bin count of 25 was used for the MR images as per the Image Biomarker Standardization Initiative guidelines [29]. Briefly, a fixed bin count introduces a normalizing effect for MR which may be beneficial when intensity units are arbitrary and allows for a direct comparison of feature values across multiple analyzed ROIs (e.g. across different samples). All textural features were normalized by subtracting the values from their mean and dividing by the standard deviation.

Major categories of extracted features included shape, first order statistics, gray level co-occurrence matrix (GLCM), gray level run-length matrix (GLRLM), and gray level size-zone matrix (GLSZM) features derived from original images as well as after wavelet filtering. The angles required for the computation of the GLCM and GLRLM were automatically generated and averaged to achieve rotational invariance. The distance for the associated angle was set to 1 voxel in all directions for the GLCM. We used 25 equally sized bins for CT and 25 fixed bin count for MR for first-order statistics, and gray levels were quantized into 20-25 levels for CT and 25 levels for MR for GLCM and GLRLM calculations. For the detailed list of features calculated, we refer readers to [25]. Wavelet filtering resulted in either a high-pass or low-pass filter in each of the three dimensions (e.g. wavelet LLL corresponded to low-pass filter applied in the $\mathrm{x}-, \mathrm{y}-$, and $\mathrm{z}$-axis directions). In broad terms, first order features describe the statistics of voxel intensity distributions within the ROI while higher order features

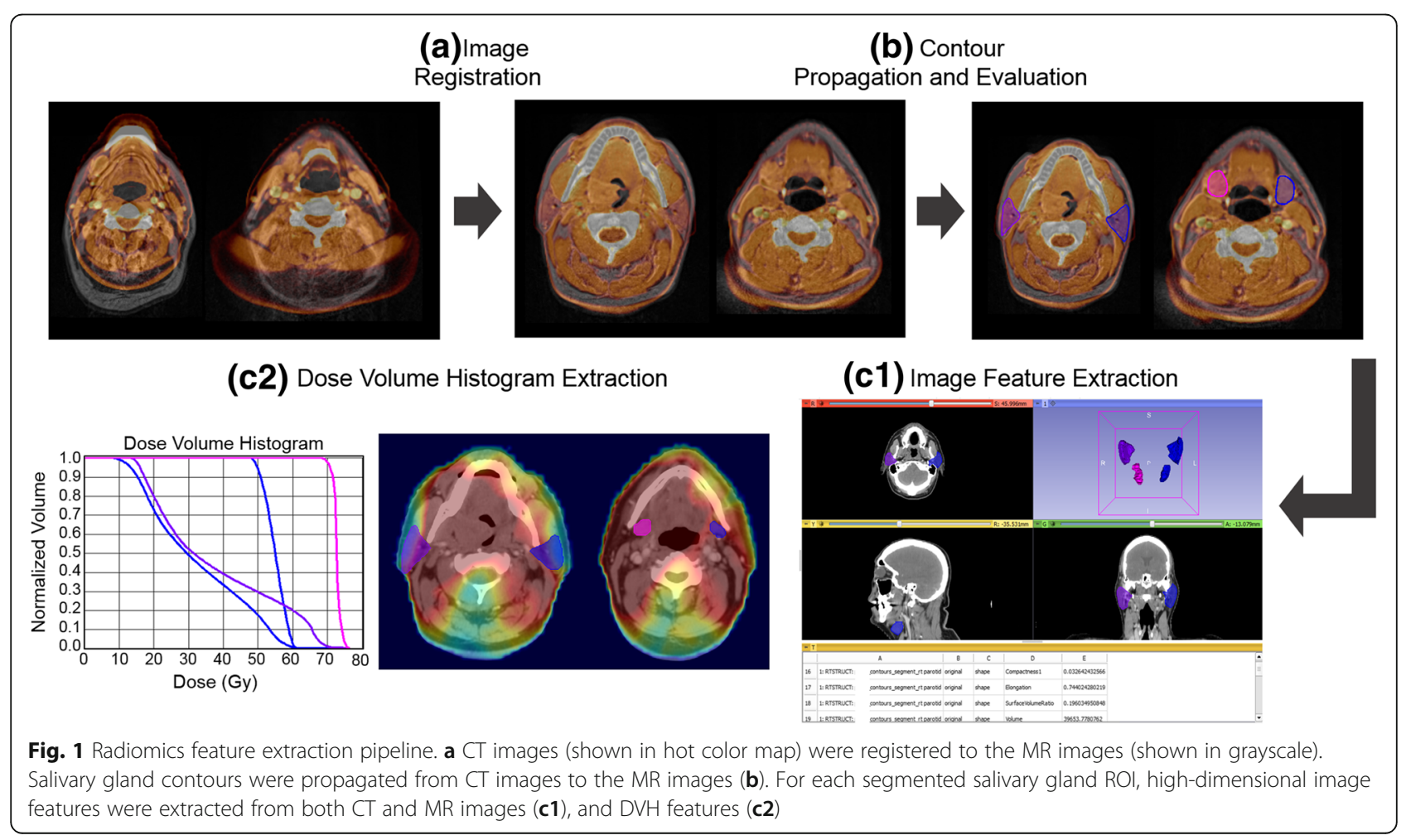


such as GLCM, GLRLM, and GLSZM seek to quantify textural characteristics. Taken together, 2877 image features and $48 \mathrm{DVH}$ features were extracted for each patient. The datasets used and analyzed during the current study are available from the corresponding author on reasonable request.

\section{Feature selection and xerostomia prediction}

As per Transparent Reporting of a multivariable prediction model for Individual Prognosis Or Diagnosis (TRIPOD) guidelines [30], a Spearman correlation was used prior to modelling to pre-select features that were correlated with the outcome $(p<0.05)$. There was no correction performed for multiple testing. This resulted in 102 image features and $44 \mathrm{DVH}$ features. A shrinkage regression analysis of the pre-selected features (including the image features, DVH information, age, gender, and tumor volume) was performed using the least absolute shrinkage and selection operator (LASSO). The internal validity of the variable selection was estimated by repeating the entire variable selection procedure using a leaveone-out-cross-validation. The most frequently selected variables $(>50 \%)$ were considered in the final model. Finally, to further address collinearity, if the correlation coefficient between two features was larger than 0.80 , only the variable with the highest correlation with xerostomia was selected, as previously described [31,32]. This resulted in one image feature to be removed (specifically, the CT contralateral submandibular wavelet LLH GLSZM Gray Level Non Uniformity Normalized). Feature selection was performed on the training set only (specifically, patients treated between the years 2009 and 2016).

Trained on the institution cohort of patients treated between the years 2009 and 2016, prediction modelling was performed using generalized linear model (GLM) with a repeated ten-fold cross validation [33] to predict radiation-induced xerostomia at 3 months after RT. The ten-fold cross validation was performed 100 times with random initialization of 10 disjoint groups. As per TRIPOD guidelines [30], in an independent set of patients who were treated in 2017 and 2018 (specifically after December 31, 2016), we compared the prediction performance under eight different scenarios: 1) only DVH features, 2) only CT image features, 3) only MR image features, 4) both CT and MR image features, 5) DVH and CT image features, 6) $\mathrm{DVH}$ and CT/MR image features, 7) clinical and CT/MR image features, and 8) clinical, DVH, CT/MR image features. Clinical data included age, sex, and tumor volume. The model performance measures were the area under the receiver operating characteristic curve (ROC-AUC). DeLong's test was used to analyze the areas under correlated ROC curves [34]. The 95\% confidence interval (CI) was computed for the AUC.

All statistical analysis and predictive modeling was performed in $\mathrm{R}$ (version 3.4.1). Results were considered significant when the probability of making a Type I error was less than $5 \%(p<0.05)$.

\section{Results}

\section{Study subjects}

Two hundred and sixty-six HNC patients were evaluated including those with and without xerostomia. Table 1 shows demographics, tumor and DVH information for all patients. Most patients had tumors of the oropharynx ( $n=119 / 266,45 \%)$ or the oral cavity $(n=23 / 266,23 \%)$. All patients were treated with IMRT, specifically, $7 \%$ of patients in the training cohort and $44 \%$ of patients in the validation cohort, received TomoTherapy.

CT and MR images of parotid glands from four representative patients are shown in Fig. 2. Briefly, patients with post-treatment xerostomia appeared to have more hypodense and heterogeneous parotid glands at baseline, compared to those without xerostomia in the CT images. Both CT and MR images of patients with xerostomia appeared to have more regions of lower intensity.

Figure 3 shows $\mathrm{CT}$ and MR images of submandibular glands for four representative patients. On CT, the

Table 1 Characteristics for head and neck cancer patients ( $n=$ 266). Continuous variables are displayed as mean (SD), while categorical variables are displayed as count (\%)

\begin{tabular}{lll}
\hline & $\begin{array}{l}\text { Internal Validation } \\
\text { Cohort } \\
(\mathrm{n}=216)\end{array}$ & $\begin{array}{l}\text { External Validation } \\
\text { Cohort } \\
(\mathrm{n}=50)\end{array}$ \\
\hline Patient Demographics & $60(13)$ \\
Age (yrs) & $58(10)$ & $46(92)$ \\
Male $n$ & $176(81)$ & $14(28)$ \\
Xerostomia $\geq 2$ & $87(40)$ & $22(44)$ \\
$\mathrm{n}$ & $16(7)$ & \\
TomoTherapy $n$ & $27(54)$ \\
Tumor Site & & $13(26)$ \\
Oropharynx & $92(43)$ & $6(12)$ \\
Oral Cavity & $49(23)$ & $0(0)$ \\
Nasopharynx & $13(6)$ & $3(6)$ \\
Hypopharynx & $4(2)$ & $0(0)$ \\
Larynx & $19(9)$ & $1(2)$ \\
Thyroid & $5(2)$ & $0(0)$ \\
Accessory & $7(3)$ & $22.8(9.9)$ \\
Sinuses & & $49.5(17.8)$ \\
Other & $27(12)$ & \\
DVH Parameters & & $27.8(14.7)$ \\
CPG D40 (Gy) & $56.8(23.5)$ & \\
cSG D60 (Gy) & & \\
\hline
\end{tabular}



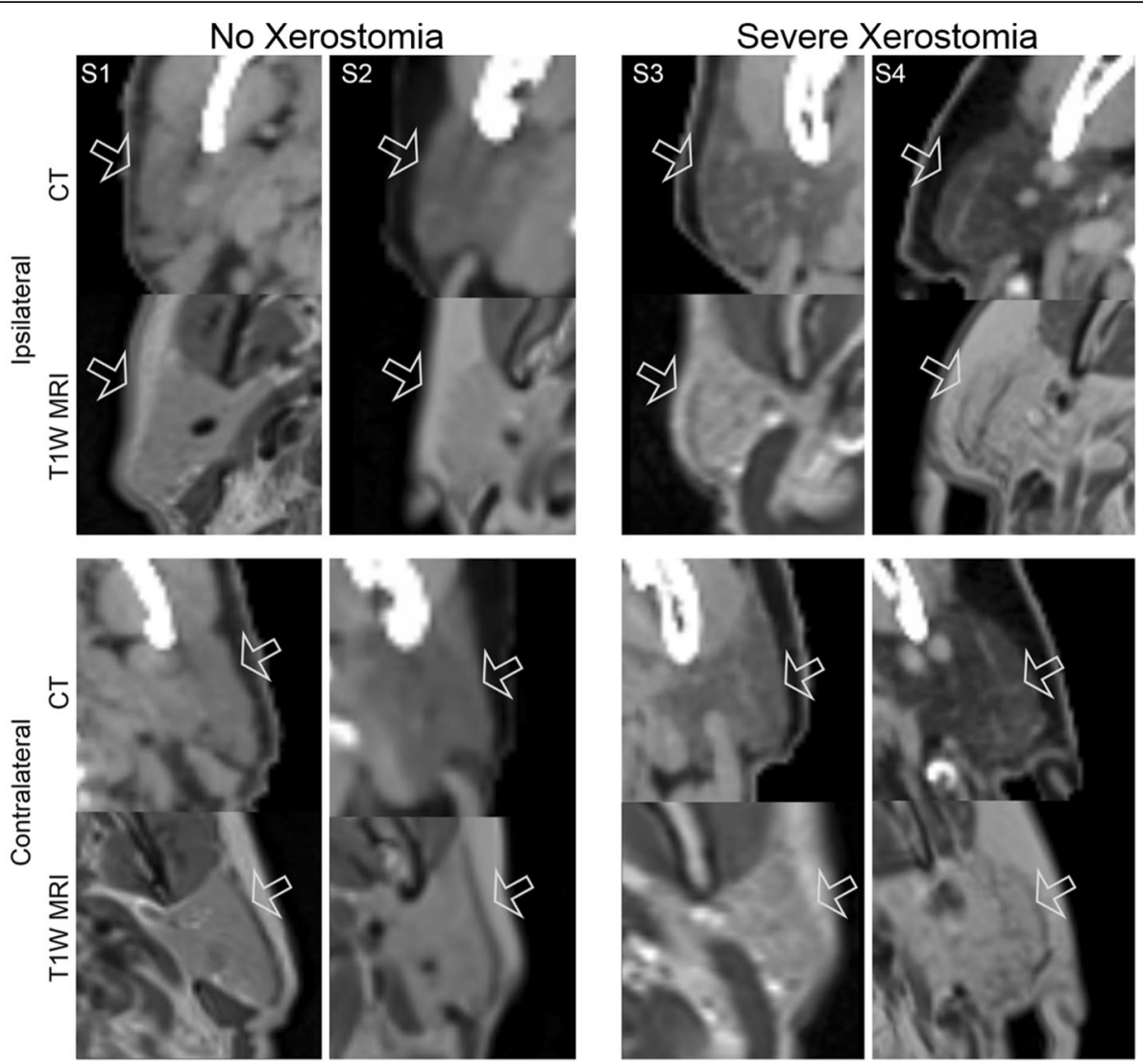

Fig. 2 CT and MR images of representative patients' parotid glands. From left to right: S1: 57-yr old male with squamous cell carcinoma of the nasopharynx, S2: 82-yr old female with melanoma of nasal cavity, S3: 61-yr old male with squamous cell carcinoma of the nasopharynx, and S4: 69-yr old male with carcinoma ex pleomorphic adenoma of eye. Images are displayed using the same window and level

submandibular glands of patients with xerostomia again appeared more hypodense and heterogeneous compared to those patients without xerostomia. The MR images of patients with xerostomia appear to be more heterogeneous and hypointense than the patients without xerostomia similarly as in CT.

\section{Xerostomia prediction models}

Table 2 shows the performance of prediction models for development of xerostomia at 3 months post-RT for the internal validation cohort. Using DeLong's test, the DVH-ROC was not significantly different from MRROC $(p=0.6)$ and CT-ROC $(p=0.6)$. The CT-ROC was not significantly different from the MR-ROC $(p=0.7)$. Combining CT with MR features suggested an improvement in xerostomia prediction performance compared to CT features alone $(p=0.01)$. Finally, the model performance improved with the combination of DVH and $\mathrm{CT}+\mathrm{MR}$ features compared to DVH $(p=0.003)$ or CT + MR $(p=0.03)$. There was a trend towards significance when comparing the DVH +CT-ROC to the DVH + $\mathrm{CT}+\mathrm{MR}-\mathrm{ROC}(p=0.06)$. The model performance improved with the combination of $\mathrm{DVH}$ and $\mathrm{CT}+\mathrm{MR}$ features compared to DVH, CT, or MR alone $(p<0.005)$. Finally, adding the clinical data did not significantly change the $\mathrm{DVH}+\mathrm{CT}+\mathrm{MR}-\mathrm{ROC}(p=0.2)$. Training set ROC curves are shown in the supplement as Additional file 1: Figure S1.

Table 3 shows the regression coefficients $(\beta)$ and odds ratios (ORs) for all the features used in the GLM prediction models. For the DVH only model, cPG D40 contributed significantly to the model $(\mathrm{OR}=1.51, p<0.001)$. For the CT only model, features that contributed significantly to the model included those from both salivary glands, and interestingly, most of the image features stemmed from the wavelet filtered images. For the MR only model, features from both the parotid and submandibular glands contributed significantly to the model including the cPG least axis $(\mathrm{OR}=2.66, p=0.004)$, iPG wavelet LHL GLSZM small area high gray level emphasis $(\mathrm{OR}=0.50, p=0.03)$, and iSG wavelet $\mathrm{LHH}$ GLSZM small area high gray level emphasis $(\mathrm{OR}=3.22$, $p=0.0005)$.

In the model containing Clinical, DVH, CT, and MR features, the features that significantly contributed to the model were the cPG D40 (OR $=2.79, p=0.04)$, CT cSG 

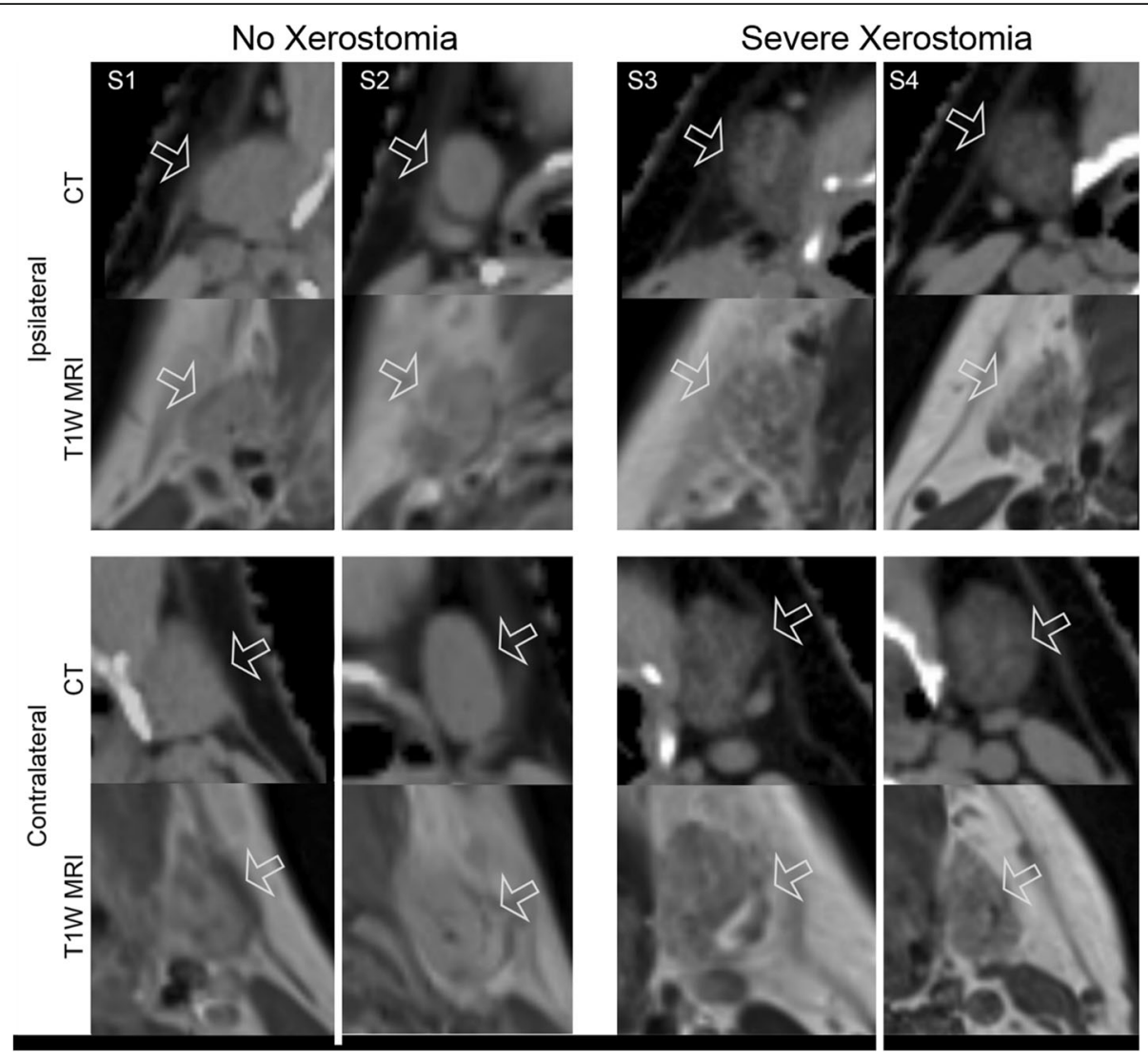

Fig. 3 CT and MR images of representative patients' submandibular glands. From left to right: S1: 71-yr old male with squamous cell carcinoma of the base of tongue, S2: 82-yr old female with melanoma of nasal cavity, S3: 54-yr old female with squamous cell carcinoma of the tonsil, and 54: 61-yr old male with squamous cell carcinoma of the nasopharynx. Images are displayed using the same window and level

Table 2 Multiple Logistic Regression performances using a 10fold cross validation at predicting xerostomia at 3 months after radiotherapy for internal validation cohort $(n=216)$. Mean and standard deviation of area-under-the curve is reported for the repeated 10 fold cross-validation

\begin{tabular}{llll}
\hline Mean \pm SD & AUC & Sensitivity & Specificity \\
\hline Generalized Linear Model & & & \\
DVH & $0.73 \pm 0.01$ & $0.76 \pm 0.01$ & $0.56 \pm 0.02$ \\
CT & $0.69 \pm 0.01$ & $0.76 \pm 0.01$ & $0.50 \pm 0.02$ \\
MR & $0.70 \pm 0.01$ & $0.80 \pm 0.01$ & $0.50 \pm 0.02$ \\
$C T+M R$ & $0.75 \pm 0.01$ & $0.76 \pm 0.01$ & $0.58 \pm 0.02$ \\
DVH +CT & $0.77 \pm 0.01$ & $0.79 \pm 0.01$ & $0.60 \pm 0.02$ \\
DVH +CT + MR & $0.79 \pm 0.01$ & $0.78 \pm 0.01$ & $0.65 \pm 0.02$ \\
Clinical+CT+MR & $0.77 \pm 0.01$ & $0.79 \pm 0.02$ & $0.61 \pm 0.02$ \\
Clinical+DVH $+C T+M R$ & $0.79 \pm 0.01$ & $0.78 \pm 0.02$ & $0.65 \pm 0.02$ \\
\hline
\end{tabular}

wavelet LLL GLSZM gray level non uniformity normalized $(\mathrm{OR}=2.29, p=0.04)$, MR iSG wavelet LHH GLSZM small area high gray level emphasis $(\mathrm{OR}=3.59, p=0.002)$, and the MR iSG wavelet LHH GLSZM gray level non uniformity normalized ( $\mathrm{OR}=0.40, p=0.04)$. Interestingly, all of the significant image features came from wavelet filtered images and stemmed from the GLSZM. Additional file 3: Table S1 shows the beta coefficients and OR for the CT + $\mathrm{MR}, \mathrm{DVH}+\mathrm{CT}, \mathrm{DVH}+\mathrm{CT}+\mathrm{MR}$, and Clinical $+\mathrm{CT}+\mathrm{MR}$ models.

Table 4 shows the performance of the prediction models for the validation cohort and ROC curves are shown in the supplement. The CT-ROC and MR-ROC were not significantly different $(p=0.4)$. The DVH-ROC was not significantly different than the CT-ROC $(p=$ $0.8)$ or MR-ROC $(p=0.4)$. However, the CT + MR-ROC was significantly different than the CT-ROC $(p=0.03)$, but not significantly different than the DVH-ROC ( $p=$ $0.4)$ or MR-ROC $(p=0.8)$. The Clinical+CT + MR ROC was significantly different than the CT-ROC (0.02), but not MR-ROC (0.2). The Clinical+DVH + CT + MR model was significantly different from Clinical $+\mathrm{CT}+\mathrm{MR}$ $(p=0.03)$, but not from the CT + MR model $(p=0.5)$. 
Table 3 GLM summary including odds ratios (OR) and 95\% confidence interval (CI) for the prediction of xerostomia

\begin{tabular}{|c|c|c|c|c|c|c|c|c|}
\hline & \multicolumn{2}{|c|}{ DVH Model } & \multicolumn{2}{|c|}{ CT Model } & \multicolumn{2}{|c|}{ MR Model } & \multicolumn{2}{|c|}{$\begin{array}{l}\text { Clinical + DVH +CT+ } \\
\text { MR Model }\end{array}$} \\
\hline & $\beta$ & $\begin{array}{l}\mathrm{OR} \\
(95 \% \mathrm{Cl})\end{array}$ & $\beta$ & $\begin{array}{l}\mathrm{OR} \\
(95 \% \mathrm{Cl})\end{array}$ & $\beta$ & $\begin{array}{l}\mathrm{OR} \\
(95 \% \mathrm{Cl})\end{array}$ & $\bar{\beta}$ & $\begin{array}{l}\mathrm{OR} \\
(95 \% \mathrm{Cl})\end{array}$ \\
\hline Intercept & -2.58 & - & -9.21 & & -0.31 & & -6.54 & \\
\hline Age & - & - & - & - & - & - & -0.69 & $\begin{array}{l}0.50(0.47- \\
0.54)\end{array}$ \\
\hline Gender & - & - & - & - & - & - & 1.09 & $\begin{array}{l}2.97(1.93- \\
4.01)\end{array}$ \\
\hline Tumor Volume & - & - & - & - & - & - & 0.45 & $\begin{array}{l}1.56(1.56- \\
1.58)\end{array}$ \\
\hline CPG D40 & 1.51 & $\begin{array}{l}4.57(4.56- \\
4.58)\end{array}$ & - & - & - & - & 1.03 & $\begin{array}{l}2.79(2.78- \\
2.79)\end{array}$ \\
\hline CSG D60 & 0.60 & $\begin{array}{l}1.81(1.80- \\
1.82)\end{array}$ & - & - & - & - & 0.63 & $\begin{array}{l}1.86(1.86- \\
1.88)\end{array}$ \\
\hline $\begin{array}{l}\text { CT cSG wavelet LLL GLSZM Gray Level Non } \\
\text { Uniformity Normalized }\end{array}$ & - & - & 1.00 & $\begin{array}{l}2.73(-11.3- \\
16.7)\end{array}$ & - & - & 0.83 & $\begin{array}{l}2.29(-14.4- \\
19.0)\end{array}$ \\
\hline $\begin{array}{l}\text { CT iPG original GLSZM Low Gray Level Zone } \\
\text { Emphasis }\end{array}$ & - & - & -0.64 & $\begin{array}{l}0.53(-9.0- \\
10.1)\end{array}$ & - & - & -0.61 & $\begin{array}{l}0.55(-10.4- \\
11.5)\end{array}$ \\
\hline CT iSG wavelet HLL GLCM Inverse Variance & - & - & 0.57 & $\begin{array}{l}1.77(-17.4- \\
20.9)\end{array}$ & - & - & 0.36 & $\begin{array}{l}1.44(-21.9- \\
24.8)\end{array}$ \\
\hline CT cPG wavelet LHL Total Energy & - & - & 0.42 & $\begin{array}{l}1.53(1.52- \\
1.54)\end{array}$ & - & - & 0.31 & $\begin{array}{l}1.36(1.35- \\
1.37)\end{array}$ \\
\hline $\begin{array}{l}\text { CT iSG wavelet HLL GLRLM Long Run High Gray Level } \\
\text { Emphasis }\end{array}$ & - & - & -0.65 & $\begin{array}{l}0.52(0.51- \\
0.53)\end{array}$ & - & - & -0.69 & $\begin{array}{l}0.50(0.49- \\
0.51)\end{array}$ \\
\hline $\mathrm{CT}$ iPG original first order 10 Percentile & - & - & -0.69 & $\begin{array}{l}0.50(0.49- \\
0.51)\end{array}$ & - & - & -0.38 & $\begin{array}{l}0.69(0.68- \\
0.70)\end{array}$ \\
\hline $\begin{array}{l}\text { CT CPG wavelet LHL GLRLM Long Run High Gray } \\
\text { Level Emphasis }\end{array}$ & - & - & 0.43 & $\begin{array}{l}1.54(1.53- \\
1.55)\end{array}$ & - & - & 0.49 & $\begin{array}{l}1.63(1.62- \\
1.64)\end{array}$ \\
\hline MR CPG shape Least Axis Length & - & - & - & - & 0.98 & $\begin{array}{l}2.66(2.59- \\
2.73)\end{array}$ & 0.84 & $\begin{array}{l}2.32(2.23- \\
2.42)\end{array}$ \\
\hline $\begin{array}{l}\text { MR iSG wavelet LHH GLSZM Gray Level Non } \\
\text { Uniformity Normalized }\end{array}$ & - & - & - & - & -0.65 & $\begin{array}{l}0.52(-26.2- \\
27.2)\end{array}$ & -0.92 & $\begin{array}{l}0.40(-33.8- \\
34.6)\end{array}$ \\
\hline $\begin{array}{l}\text { MR iSG wavelet LHH GLSZM Small Area High Gray } \\
\text { Level Emphasis }\end{array}$ & - & - & - & - & 1.17 & $\begin{array}{l}3.22(3.20- \\
3.23)\end{array}$ & 1.28 & $\begin{array}{l}3.59(3.57- \\
3.61)\end{array}$ \\
\hline $\begin{array}{l}\text { MR iPG wavelet LHL GLSZM Small Area High Gray } \\
\text { Level Emphasis }\end{array}$ & - & - & - & - & -0.69 & $\begin{array}{l}0.50(0.48- \\
0.52)\end{array}$ & -0.72 & $\begin{array}{l}0.49(0.47- \\
0.51)\end{array}$ \\
\hline $\begin{array}{l}\text { MR iSG wavelet LLH GLSZM Size Zone Non } \\
\text { Uniformity Normalized }\end{array}$ & - & - & - & - & -0.36 & $\begin{array}{l}0.70(-9.75- \\
11.2)\end{array}$ & -0.43 & $\begin{array}{l}0.65(-12.3- \\
13.6)\end{array}$ \\
\hline
\end{tabular}

iPG ipsilateral parotid gland, CPG contralateral parotid gland, iSG ipsilateral submandibular gland, $c S G$ contralateral submandibular gland, GLCM gray level cooccurrence matrix, GLSZM gray level size zone matrix, GLRLM gray level run length matrix; bold indicates significant values $(p<.05)$

Adding clinical data to the $\mathrm{DVH}+\mathrm{CT}+\mathrm{MR}$ model, modestly improved the model performance with a trend towards significant $(p=0.1)$. Test set ROC curves are shown in the supplement as Additional file 2: Figure S2.

\section{Discussion}

In this study, to better understand the influence of image features in the prediction of RT-induced xerostomia, we investigated the relationships between $\mathrm{CT}$ and $\mathrm{MR}$ image features with xerostomia scores in HNC patients using machine learning approaches. We made the following observations: 1) image features from both the parotid and submandibular glands significantly contributed to our prediction of xerostomia, 2) higher order texture features for both ipsi- and contralateral salivary glands were important predictors of xerostomia, and 3) combining multimodal image features with dosimetry features improved xerostomia prediction. Collectively, these observations further support prior work [22, 35] demonstrating that baseline salivary gland image features with $\mathrm{CT}$ along with quantifying radiation injury are important in predicting for the risk of xerostomia 3 months following RT.

mage features from both salivary glands significantly contributed to the prediction of xerostomia post-RT, concordant with the readily apparent differences 
Table 4 Multiple Logistic Regression performances at predicting xerostomia at 3 months after radiotherapy for external validation cohort $(n=50)$

\begin{tabular}{llll}
\hline & AUC & Sensitivity & Specificity \\
\hline Generalized Linear Model & & & \\
DVH & $0.63(0.51-0.81)$ & 0.64 & 0.58 \\
CT & $0.57(0.45-0.71)$ & 0.50 & 0.68 \\
MR & $0.66(0.54-0.82)$ & 0.80 & 0.65 \\
CT + MR & $0.70(0.57-0.82)$ & 0.80 & 0.62 \\
DVH +CT & $0.56(0.40-0.68)$ & 0.60 & 0.56 \\
DVH +CT + MR & $0.60(0.50-0.73)$ & 0.67 & 0.53 \\
Clinical+CT + MR & $0.73(0.62-0.86)$ & 0.86 & 0.59 \\
Clinical+DVH + CT + MR & $0.68(0.52-0.80)$ & 0.67 & 0.68 \\
\hline
\end{tabular}

visualized in both salivary glands using $\mathrm{CT}$ and $\mathrm{MR}$ (Figs. 2 and 3). Patients with xerostomia after RT appeared to have more heterogeneous parotid and submandibular glands at baseline. We should note that the majority of $\mathrm{HNC}$ research using radiomics has focused on the parotid glands [31, 36-39] with relatively little attention paid to the submandibular glands $[18,22]$. Interestingly, the features with the greatest OR corresponded to the submandibular glands. While the parotid glands produce the majority of saliva during eating and with oral stimulation, submandibular glands contribute up to more than $70 \%$ of unstimulated/resting salivary output [40] which is rich in mucin. This allows for the oral mucosa to maintain its hydration $[41,42]$. These results suggest that baseline submandibular gland image features may provide insight into unstimulated salivary function, and this insight may improve prediction of susceptibility to post-RT xerostomia.

Important features in our cohort stemmed from the GLRLM and the GLSZM and both the ipsilateral and contralateral salivary glands. For the contralateral side, the CT SG wavelet LLL GLRLM gray level nonuniformity normalized significantly contributed to the GLM. The cPG CT wavelet LHL GLRLM long run high gray level emphasis, which had the second lowest standard error in the model, increases when the texture is dominated by long runs with high intensity levels. These results suggest that patients with xerostomia have cSG that have lower similarity in intensities (increased gray level non-uniformity) and more heterogeneous size zone volumes (increased size zone non-uniformity). Furthermore, patients with increased risk of xerostomia have finer structural textures of the CPG (decreased long run emphasis) [22] with longer run of high intensity voxels (increased long run high gray level emphasis). Focusing on the ipsilateral side, the feature that contributed significantly to the GLM included the MR iSG wavelet LHL GLSZM small area high gray level emphasis. This feature indicates that patients with xerostomia have ipsilateral submandibular glands with more small regions of low intensity (i.e. more locally heterogeneous as indicated by an increase of small area low gray level intensity).

Similar to previously reported work, these image features suggest that patients who are likely to develop xerostomia have more locally heterogeneous salivary glands. The heterogeneity differences can be seen in the representative images (Figs. 2 and 3) where patients with xerostomia had more regions of low intensities in both parotid and submandibular glands compared to those patients without xerostomia. This is consistent with previously published work demonstrating that patients who develop xerostomia after RT have more heterogeneous parotid gland tissue [22]. More recently, MR derived image features of the parotid glands were used in the prediction of xerostomia in HNC patients [31]. This important work demonstrated that high signal intensity, specifically the 90th percentile of the MR-intensities in parotid glands improved the performance of the xerostomia prediction model. It is well known that high signal intensity in T1-weighted images is related to fat deposition because of the short $\mathrm{T} 1$ relaxation time of fatty tissue [43]. In fact, fat deposition may represent the loss of normal glandular cells as this phenomenon is also seen in diseases such as Sjögren's syndrome which is characterized by autoimmune destruction of salivary and lacrimal glands [44]. Of note, the salivary glands of patients with Sjögren's syndrome have also been shown to be more heterogeneous than those without this syndrome [45]. Fatty replaced salivary glands have also been shown to be related to age [46] and xerostomia [47]. However, in our cohort, age was not correlated with image features or xerostomia. On CT, fatty tissue appears as low density [48]. This is consistent with the representative CT images (Figs. 2 and 3) of the parotid and submandibular glands, where the patients with xerostomia had hypodense salivary glands (with obvious local heterogeneous regions).

Finally, in our xerostomia prediction models, for our training cohort, there were no significant differences between our DVH, CT-only, and MR-only models. However, when $\mathrm{CT}$ and MR were combined, the performance improved compared to DVH alone. More importantly, we observed that the combination of dosimetry and image features improved overall prediction compared to dosimetry or image features alone. However, the specificity of our models with CT, MR, and DVH-only features was low. In fact, the combination of $\mathrm{DVH}+\mathrm{CT}+\mathrm{MR}$ features did not lead to a significant improvement in sensitivity and specificity. With the addition of all features in a single model, the sensitivity improved only modestly. We should note that majority of our patients 
did not develop xerostomia, resulting in a biased dataset which could influence sensitivity and specificity. Compared to previously published work evaluating $\mathrm{CT}$ image features to predict xerostomia at 12 months [22], the performance of our models was comparable. This work reported an AUC of 0.77 with the inclusion of CT features, specifically features derived from the GLRLM and GLSZM. Other work that has used imaging to predict xerostomia at 12 months using CT only [37, 38] and MR only [31] parotid gland image features has also demonstrated comparable performance to our models (AUC range: $0.60-0.80$ ). Cone beam CT of the parotid glands has also been used to predict xerostomia in a single cohort with performance ranging from 0.71-0.76 [49]. Other work that has used CT parotid image features with dosimetry in a single cohort with nested cross validation has also shown model performances in the range of $0.68-0.88$ [50]. In our validation cohort, we observed a similar trend where combining imaging improved performance. Adding dosimetry to our training cohort did improve performance which is consistent with previously published work that has shown the prediction of xerostomia improves when CT image features are combined with dosimetric information [18, 22, 35, 38]. However, in our validation set, adding dosimetry to imaging features did not improve performance. It should be noted that our work used time to separate our training and validation sets. The decrease in performance of the DVH model may be indicative of evolving practices of the attending physicians. Specifically, changes in physician preferences of dose constraints to the salivary glands. The reduction in performance may also reflect limitations of the DVH in capturing 3D spatial information. This may also explain the decrease in performance of the validation models that contained DVH features. It should also be noted that combining clinical data with CT and MR significantly improved xerostomia prediction compared to CT alone. Although the receiver operating characteristic curves had overlapping confidence intervals, there was a trend towards prediction improvement when combining clinical data with dosimetry and image features compared to dosimetry and CT features alone which to our knowledge has not been previously demonstrated. Future work in an independent dataset is required to further determine the benefits of combining imaging modalities in outcome prediction modelling.

Although this study provides promising preliminary results, future work is needed to ascertain the generalizability of these findings. It should be noted that random variation in small datasets can often be mistakenly interpreted as meaningful (i.e. overfitting), and as a consequence the model may not perform as well in independent datasets. In the present work, the risks of overfitting the model were addressed by pre-selecting variables based on their inter-correlation (with no correction for multiple comparisons since $p$-values at this step were simply used to selected a group of candidate features which were further refined using LASSO), cross-validation of the internal dataset, and validating our models using a temporally split dataset [30]. It should be noted that temporal splitting is an intermediate validation method compared to internal and external validation [30]. Future work will need to validate these models on an independent external dataset. The presence of multiple correlated explanatory variables can lead to unstable models with highly variable coefficient estimates and incorrect selection of significant texture features. In this work, collinearity was addressed by determining the Pearson correlation coefficient between two features [30-32]. If the correlation coefficient was larger than 0.80 , only the variable with the highest correlation with xerostomia was selected. Modality specific resampling was not performed for the CT images. and non-cubic voxels were used for radiomics analysis, similar to prior studies [18, 22, 31, 38]. Resampling images compared to using the original resolution before feature computation is an active area of radiomics research, and there is no widely accepted recommendation. Resampling images to an isotropic resolution may lead to better interpretation of certain features, but there will be information loss/degradation due to interpolation process. For our MR images, we used the same scanning protocol for training and validation. This may limit the translatability and generalizability of our results because MR intensities are highly dependent on scanning protocol. Also, unlike CT, MR signal-intensity is influenced by hardware factors such as the positioning of the RF coils, which introduce inter-scan variability. Although normalization of MR data has been proposed to address this, the benefits of normalization for radiomic prediction models to differentiate patients with or without xerostomia has not been well established. Future work is needed to establish the benefits of signal normalization for radiomic prediction models of xerostomia. In our work, salivary glands were contoured by the patient's attending radiation oncologist or by multi-atlas-based auto-segmentation with manual assessment/correction (when clinical contours were not available). Although multiple observers did not contour the same patient, multiple observers' contours of the glands were included in our feature selection and prediction model building process. Therefore, we anticipate that the selected features are robust to contour variability while being relevant to the outcome. Although previous studies have shown that inter-observer delineation variability has a relevant influence on radiomics analysis [22, 51], we should note that it is important to determine a model that is robust to variability in raw clinically available data so that it can be used in a real clinical scenario. However, further study will be needed to better understand 
the influence of contour variability to the computed radiomics features and successive feature selectionprediction performance. Finally, we acknowledge that our image feature analysis was limited to a single bin size for CT and single bin count for MRI. Texture features have been shown to be affected by the bin width or number of bins used to discretize image intensities. Although the optimal bin width/count for image feature analysis has not been established, previous HNC work has used a 25 unit bin width (similar to the bin width we used) for the evaluation of image features [27, 28]. However, since image features depend on the way they are computed (i.e. using different binning strategies) further work is needed to investigate the dependency of bin width and the selection of image features on xerostomia prediction.

\section{Conclusions}

This study suggests that baseline image features stemming from both the parotid and submandibular glands have the potential to be used as a clinical surrogate for baseline function. Features from the submandibular glands, specifically, may provide insight into unstimulated salivary function thus providing an improved prediction of susceptibility to post-RT xerostomia. Although there was a trend towards prediction improvement when all data was combined, future work is required to further determine the benefits of combining imaging modalities in xerostomia prediction. Taken together, prediction models based on these features can further our understanding of the development of radiation-induced xerostomia and allow us to develop patient-specific adaptations to radiation treatment plans to minimize toxicity.

\section{Additional files}

Additional file 1: Figure S1. Training set receiver operating characteristic (ROC) curves shown for: a) DVH Model, d) CT Model, c) MR Model, d) CT + MR Model, e) DVH + CT Model, f) DVH + CT + MRI Model, g) Clinical+CT + MR Model, and h) Clinical+DVH + CT + MR Model. Gray identifies the 95\% confidence interval (CI). (TIF $449 \mathrm{~kb}$ )

Additional file 2: Figure S2. Test set receiver operating characteristic (ROC) curves shown for: a) DVH Model, d) CT Model, c) MR Model, d) CT + MR Model, e) DVH + CT Model, f) DVH + CT + MRI Model, g) Clinical+CT + MR Model, and h) Clinical+DVH + CT + MR Model. Gray identifies the 95\% confidence interval (CI). (TIF $407 \mathrm{~kb}$ )

Additional file 3: Table S1. GLM summary including odds ratios (OR) and $95 \%$ confidence interval $(\mathrm{Cl})$ for the prediction of xerostomia. (DOCX $21 \mathrm{~kb}$ )

\section{Acknowledgements}

We would like to acknowledge support from the American Society for Radiation Oncology and the American Association of Physicists in Medicine through the ASTRO-AAPM Physics Resident/Post-Doctoral Fellow Seed Grant. This work was also supported by Johns Hopkins Radiation Oncology Discovery Award and Canon Medical Systems Corp.

\section{Authors' contributions}

All authors contributed to the study design and review of the manuscript. KS and $\mathrm{JL}$ were responsible for data collection, data analysis, statistical analysis, data interpretation, and writing of the manuscript. SHL and PH contributed to the statistical analysis. ZC and PL contributed for collecting data. LP contributed to clinical interpretation. TRM and HQ contributed to data interpretation. All authors read and approved the final manuscript.

\section{Funding}

Dr. Sheikh reports support from the American Society for Radiation Oncology and the American Association of Physicists in Medicine through the ASTRO-AAPM Physics Resident/Post- Doctoral Fellow Seed Grant. Dr. Lee and Dr. Quon report support from Johns Hopkins Radiation Oncology Discovery Award.

Dr. Lee and Dr. McNutt report support from Canon Medical Systems Corp.

\section{Availability of data and materials}

The datasets used and analyzed during the current study are available from the corresponding author on reasonable request.

Ethics approval and consent to participate

This study was approved by the Johns Hopkins University institutional review board.

\section{Consent for publication}

Not applicable.

\section{Competing interests}

The authors declare that they have no competing interests.

Received: 2 November 2018 Accepted: 17 July 2019

Published online: 29 July 2019

\section{References}

1. Jensen $A B$, Hansen $O$, Jorgensen $K$, Bastholt L. Influence of late side-effects upon daily life after radiotherapy for laryngeal and pharyngeal cancer. Acta Oncol. 1994;33(5):487-91.

2. Braam PM, Roesink JM, Moerland MA, Raaijmakers CPJ, Schipper M, Terhaard $\mathrm{CHJ}$. Long-term parotid gland function after radiotherapy. Int J Radiat Oncol Biol Phys. 2005;62(3):659-64

3. Pinna R, Campus G, Cumbo E, Mura I, Milia E. Xerostomia induced by radiotherapy: an overview of the physiopathology, clinical evidence, and management of the oral damage. Ther Clin Risk Manag. 2015;11:171-88.

4. Burlage FR, Coppes RP, Meertens H, Stokman MA, Vissink A. Parotid and submandibular/sublingual salivary flow during high dose radiotherapy. Radiother Oncol. 2001;61(3):271-4.

5. Lambin P, Roelofs E, Reymen B, Velazquez ER, Buijsen J, Zegers CML, et al. "Rapid Learning health care in oncology" - An approach towards decision support systems enabling customised radiotherapy. Radiother Oncol. 2013; 109(1):159-64.

6. McNutt TR, Benedict SH, Low DA, Moore K, Shpitser I, Jiang W, et al. Using big data analytics to advance precision radiation oncology. Int J Radiat Oncol Biol Phys. 2018;101(2):285-91.

7. Feng $M$, Valdes $G$, Dixit N, Solberg TD. Machine learning in radiation oncology: opportunities, requirements, and needs. Front Oncol. 2018;8:1-7. Available from: http://journal.frontiersin.org/article/10.3389/fonc.2018.00110/ full

8. Boon I, Au Yong T, Boon C. Assessing the Role of Artificial Intelligence (Al) in Clinical Oncology: Utility of Machine Learning in Radiotherapy Target Volume Delineation. Med. 2018;5(4).

9. Men $\mathrm{K}$, Zhang $\mathrm{T}$, Chen $\mathrm{X}$, Chen $\mathrm{B}$, Tang $\mathrm{Y}$, Wang $\mathrm{S}$, et al. Fully automatic and robust segmentation of the clinical target volume for radiotherapy of breast cancer using big data and deep learning. Phys Medica. 2018;50:13-9.

10. Ma M, Kovalchuk N, Buyyounouski MK, Xing L, Yang Y. Dosimetric featuresdriven machine learning model for DVH prediction in VMAT treatment planning. Med Phys. 2019;46(2):857-67.

11. Kearney V, Chan JW, Valdes G, Solberg TD, Yom SS. The application of artificial intelligence in the IMRT planning process for head and neck cancer. Oral Oncol. 2018:87:111-6.

12. Nyflot MJ, Thammasorn P, Wootton LS, Ford EC, Chaovalitwongse WA. Deep learning for patient-specific quality assurance: Identifying errors in 
radiotherapy delivery by radiomic analysis of gamma images with convolutional neural networks. 2019;46(2):456-64.

13. Cheng Z, Nakatsugawa M, Hu C, Robertson SP, Hui X, Moore JA, et al. Evaluation of classification and regression tree (CART) model in weight loss prediction following head and neck cancer radiation. Adv Radiat Oncol. 2018;3(3):346-55.

14. El Naqa I, Kerns SL, Coates J, Luo Y. Radiogenomics and radiotherapy response modeling. Phys Med Biol. 2017;62:R179-206.

15. Schlett CL, Hendel T, Weckbach S, Reiser M, Kauczor HU, Nikolaou K, et al. Population-Based Imaging and Radiomics : Rationale and Perspective of the German National Cohort MRI Study. Rofo. 2016;188(7):652-61.

16. Kickingereder P, Götz M, Muschelli J, Wick A, Neuberger U, Shinohara RT, et al. Large-scale radiomic profiling of recurrent glioblastoma identifies an imaging predictor for stratifying anti-angiogenic treatment response. Clin Cancer Res. 2016;22(23):5765-71.

17. Bar, Yaniv, Diamant, Idit, Wolf, Lior, Greenspan H. Deep learning with nonmedical training used for chest pathology identification. In: In Medical Imaging 2015: Computer-Aided Diagnosis, vol 9414. 2015. p. $94140 \mathrm{~V}$.

18. Nardone V, Tini P, Nioche C, Mazzei MA, Carfagno T, Battaglia G, et al. Texture analysis as a predictor of radiation-induced xerostomia in head and neck patients undergoing IMRT. Radiol Medica. 2018;(0123456789):1-9.

19. Ou D, Blanchard P, Rosellini S, Levy A, Nguyen F, Leijenaar RTH, et al. Predictive and prognostic value of $\mathrm{CT}$ based radiomics signature in locally advanced head and neck cancers patients treated with concurrent chemoradiotherapy or bioradiotherapy and its added value to human papillomavirus status. Oral Oncol. 2017;71:150-55.

20. Liu J, Mao Y, Li Z, Zhang D, Zhang Z, Hao S, et al. Use of texture analysis based on contrast-enhanced MRI to predict treatment response to chemoradiotherapy in nasopharyngeal carcinoma. J Magn Reson Imaging. 2016;44(2):445-55.

21. Zhai T, Dijk LV Van, Huang B, Lin Z, Ribeiro CO, Brouwer CL, et al. Improving the prediction of overall survival for head and neck cancer patients using image biomarkers in combination with clinical parameters. Radiother Oncol. 2017;124(2):256-62.

22. Dijk LV Van, Brouwer CL, Schaaf A Van Der, Burgerhof JGM, Beukinga RJ, Langendijk JA, et al. CT image biomarkers to improve patient-specific prediction of radiation- induced xerostomia and sticky saliva. Radiother Oncol. 2017:122:185-91.

23. Park S, Plishker W, Quon H, Wong J, Shekhar R, Lee J. Deformable registration of $\mathrm{CT}$ and cone-beam $\mathrm{CT}$ with local intensity matching. Phys Med Biol. 2017;62(3):927-47.

24. Asman AJ, Landman BA. Non-local statistical label fusion for multi-atlas segmentation. Med Image Anal. 2013;17(2):194-208.

25. Griethuysen JJM Van, Fedorov A, Parmar C, Hosny A, Aucoin N, Narayan V, et al. Computational Radiomics System to Decode the Radiographic Phenotype. Cancer Res. 2017;77(21):e104-e107.

26. Sha M, Zhang GG, Lati $K$. Intrinsic dependencies of $C T$ radiomic features on voxel size and number of gray levels. Med Phys. 2017:44(3):1050-62.

27. Thor M, Tyagi N, Hatzoglou V, Apte A, Saleh Z, Riaz N. A magnetic resonance imaging-based approach to quantify radiation-induced normal tissue injuries applied to trismus in head and neck cancer. Phys Imaging Radiat Oncol. 2017:34-40.

28. Brakenhoff RH, Riesterer O, Tanadini-lang S, Guckenberger M, Ikenberg K, Lambin P. Development and validation of a radiomic signature to predict HPV ( p16) status from standard CT imaging : a multicenter study. 2018;(January):1-8.

29. Alex Zwanenburg, Stefan Leger, Martin Vallières SL. Image biomarker standardisation initiative. Reference manual. 2016;

30. Collins GS, Reitsma JB, Altman DG, Moons KGM. Transparent Reporting of a multivariable prediction model for Individual Prognosis Or Diagnosis ( TRIPOD): The TRIPOD Statement. Ann Intern Med. 2015;162(1).

31. van Dijk LV, Thor M, Steenbakkers RJ, Apte A, Zhai T-T, Borra R, et al. Parotid gland fat related Magnetic Resonance Image biomarkers improve prediction of late radiation-induced xerostomia. Radiother Oncol. 2018;128(3):459-66.

32. Matzner-Lober $E$, Suehs CM, Dohan A, Molinari N. Thoughts on entering correlated imaging variables into a multivariable model: application to radiomics and texture. Diagn Interv Imaging. 2018;99(5):269-70.

33. Casella G, Fienberg S, Olkin I. Springer Texts in Statistics. Vol. 102. 2006. 618 p.

34. Delong ER, Carolina N. Comparing the areas under two or more correlated receiver operating characteristic curves : a nonparametric approach. Biometrics. 1988:44(3):837-45.
35. Nakatsugawa M, Cheng Z, Goatman K, Lee J, Robinson A, Kiess A, et al. Radiomic analysis of salivary glands for the prediction of weight loss in irradiated head and neck cancer patients. In: The 102nd Scientific Assembly and Annual Meeting of Radiological Society of North America (RSNA). 2016.

36. van Dijk L V, Brouwer CL, Laan HP Van Der, Burgerhof JGM, Langendijk JA, Steenbakkers RJHM, et al. Geometric Image Biomarker Changes of the Parotid Gland Are Associated With Late Xerostomia. Int J Radiat Oncol Biol Phys. 2017;99(5):1101-10.

37. Scalco E, Fiorino C, Mauro G, Sanguineti G, Rizzo G. Texture analysis for the assessment of structural changes in parotid glands induced by radiotherapy. Radiother Oncol. 2013:109(3):384-87.

38. Pota M, Scalco E, Sanguineti G, Farneti A, Mauro G, Rizzo G, et al. Early prediction of radiotherapy-induced parotid shrinkage and toxicity based on CT radiomics and fuzzy classification. Artif Intell Med. 2017;81:41-53.

39. Wu H, Chen X, Yang X, Tao Y, Xia Y, Deng X, et al. Early prediction of acute xerostomia during radiation therapy for head and neck Cancer based on texture analysis of daily CT. Radiat Oncol Biol. 2018;1-11.

40. Myers EN, Ferris RL. Salivary gland disorders. Pittsburg, PA: Springer; 2007. 186 p.

41. Proctor GB. The physiology of salivary secretion. 2016;70:11-25.

42. Lavelle C. Applied physiology of the mouth. Bristol: John Wright and Sons; 1975. p. $145-80$.

43. Delikatny EJ, Chawla S, Leung DJ, Poptani H. MR-visible lipids and the tumor microenvironment. NMR Biomed. 2011;24(6):592-611.

44. Izumi M, Eguchi K, Nakamura H, Nagataki S, Nakamura T. Premature Fat Deposition in the Salivary Glands Associated with Sjo " gren Syndrome : MR and CT Evidence. AJNR Am J Neuroradiol. 1997;18(5):951-8

45. Kojima I, Sakamoto M, likubo M, Kumamoto H, Muroi A, Sugawara Y, et al. Diagnostic performance of MR imaging of three major salivary glands for Sjögren's syndrome. Oral Dis. 2017;23(1):84-90.

46. Martinez-Madrigal F, Micheau C. Histology of the major salivary glands. Vol. 13, The American journal of surgical pathology. 1989. p. 879-99.

47. Orellana MF, Lagravère MO, Boychuk DGJ, Major PW, Flores-Mir C. Prevalence of xerostomia in population-based samples: a systematic review. J Public Health Dent. 2006;66(2):152-8.

48. Hemmingsson A, Johansson A, Rauschning W. Attenuation in human muscle and fat tissue in vivo and in vitro. Acta radiol. 1982;23(2).

49. Rosen BS, Hawkins PG, Polan DF, Balter JM, Brock KK, Kamp JD, et al. Early Changes in Serial CBCT-Measured Parotid Gland Biomarkers Predict Chronic Xerostomia After Head and Neck Radiation Therapy. Radiat Oncol Biol. 2018; 102(4):1319-29.

50. Gabry HS, Bangert M, Bangert M. Design and Selection of Machine Learning Methods Using Radiomics and Dosiomics for Normal Tissue Complication Probability Modeling of Xerostomia. Front Oncol. 2018;8:35.

51. Pavic M, Bogowicz M, Würms X, Glatz S, Finazzi T, Riesterer $O$, et al. Influence of inter-observer delineation variability on radiomics stability in different tumor sites. Acta Oncol. 2018:57(8):1070-4.

\section{Publisher's Note}

Springer Nature remains neutral with regard to jurisdictional claims in published maps and institutional affiliations.

Ready to submit your research? Choose BMC and benefit from:

- fast, convenient online submission

- thorough peer review by experienced researchers in your field

- rapid publication on acceptance

- support for research data, including large and complex data types

- gold Open Access which fosters wider collaboration and increased citations

- maximum visibility for your research: over $100 \mathrm{M}$ website views per year

At BMC, research is always in progress.

Learn more biomedcentral.com/submissions 